\title{
Patella Infera in an HIV Positive Patient Following Total Knee Arthroplasty
}

\author{
Georg Matziolis ${ }^{*}, 1$, Lars Morawietz $^{2}$, Doerte Matziolis ${ }^{1}$ and Carsten Perka ${ }^{1}$ \\ 'Orthopaedic Department, Center for Musculoskeletal Surgery, Berlin, Germany' \\ ${ }^{2}$ Institute for Pathology, Charité - University Medicine Berlin, Germany
}

\begin{abstract}
A lowering of the patella after total knee arthroplasty is accompanied by pain and a restriction of the range of motion. With its etiology being unclear at present, a multifactorial genesis is under discussion. For the first time, we present a case report describing an HIV infection as a possible cause of patella infera.

A 54-year-old HIV-positive woman developed patella infera (Insall-Salvati ratio 0.6) with a painful restriction of motion 18 months after implantation of a bicondylar surface prosthesis. By changing to a partially coupled endoprosthesis, distalization of the joint line and partial patellar resection, the patient was free of symptoms with a $\mathrm{ROM}$ of $0^{\circ} / 0^{\circ} / 110^{\circ}$.

All risk factors known from the literature could be excluded in this case, so that the presence of an HIV infection has to be discussed as a possible cause.
\end{abstract}

Keywords: Total knee arthroplasty, human immunodeficiency virus, patella infera.

\section{INTRODUCTION}

The reasons for the development of patella infera after total knee arthroplasty are still unclear, despite a prevalence of $25 \%[1,2]$. Verified risk factors include previous operations on the knee $[3,4]$, delayed postoperative mobilization [5] and lateral release [2].

The presence of an HIV infection is accompanied by impaired wound healing and scar formation [6], and coincides with Dupuytren's contractures [7, 8]. However, there have been no reports of an influence of HIV infection on ligamentous structures of the knee to date. For the first time, we describe a lowering of the patella in an HIVpositive woman.

\section{CASE REPORT}

The right knee of a 54-year-old woman was treated with a cementless bicondylar surface prosthesis without patellar replacement, due to primary varus gonarthrosis in January 1999 (Foundation Knee, Endoplus, Germany). The patient did not have an orthopedic or internal medical history. The endoprosthesis was correctly positioned, in particular the preoperative joint line was maintained with an Insall-Salvati ratio of 1.1. Computed tomography was used to ensure the correct rotation of the femoral and tibial components. Lateral release was not necessary on primary implantation. The patient was discharged after a complication-free course, with a ROM of $0^{\circ} / 0^{\circ} / 90^{\circ}$.

18 months later, the patient presented with a painful restriction of motion to $0^{\circ} / 15^{\circ} / 70^{\circ}$. Clinical symptoms were

\footnotetext{
*Address correspondence to this author at the Orthopaedic Department, Center for Musculoskeletal Surgery, Charité - University Medicine Berlin, Charitéplatz 1, D-10117 Berlin, Germany; Tel: +49 - 30 - 450 615093; Fax: +49-30-450 515922; E-mail: Georg.Matziolis@Charite.de
}

anterior knee pain, a mild overheating of the joint, and crepitation over the entire range of motion. Plain radiographs revealed a marked patella infera (Insal-Salvati ratio 0.6). Radiological controls conducted over the further course showed a successive lowering of the patella (Fig. 1). The bone mineral density of the patella appeared to be normal in the plain radiographs, so that reflex dystrophy and at least markedly deficient blood supply to the patella and surrounding tissue could be ruled out. In the laboratory tests, an HIV infection was detected for the first time. The patient was completely asymptomatic.

Revision surgery was performed, with the prosthesis being changed to a partially coupled system (Solution, Endoplus, Germany). Intraoperatively, scar changes of the thickened and shortened patellar ligament were seen, with a normal Hoffa's body. A biopsy of the patellar ligament revealed degenerative changes, with a reduction in cell density and compression of collagen fibers (Fig. 2). The tibial and femoral components were correctly rotated and the patella was centered.

The joint line was distalized $8 \mathrm{~mm}$ and the patella was partially resected $8 \mathrm{~mm}$ distally (Fig. 3). The range of motion under anesthesia was improved from $0^{\circ} / 15^{\circ} / 70^{\circ}$ to $0^{\circ} / 0^{\circ} / 120^{\circ}$. The postoperative course was free of complications, so that the patient could be discharged with an active $\mathrm{ROM}$ of $0^{\circ} 0^{\circ} / 110^{\circ}$ and complete freedom from pain.

\section{DISCUSSION}

The etiology of patella infera remains unclear and is a matter of controversial debate. The risk factors verified to date include previous repositioning osteotomies [3, 4], prolonged postoperative immobilization [5] and the performance of lateral release [2]. Weale concludes that lateral release, by reducing the blood supply to the patellar 


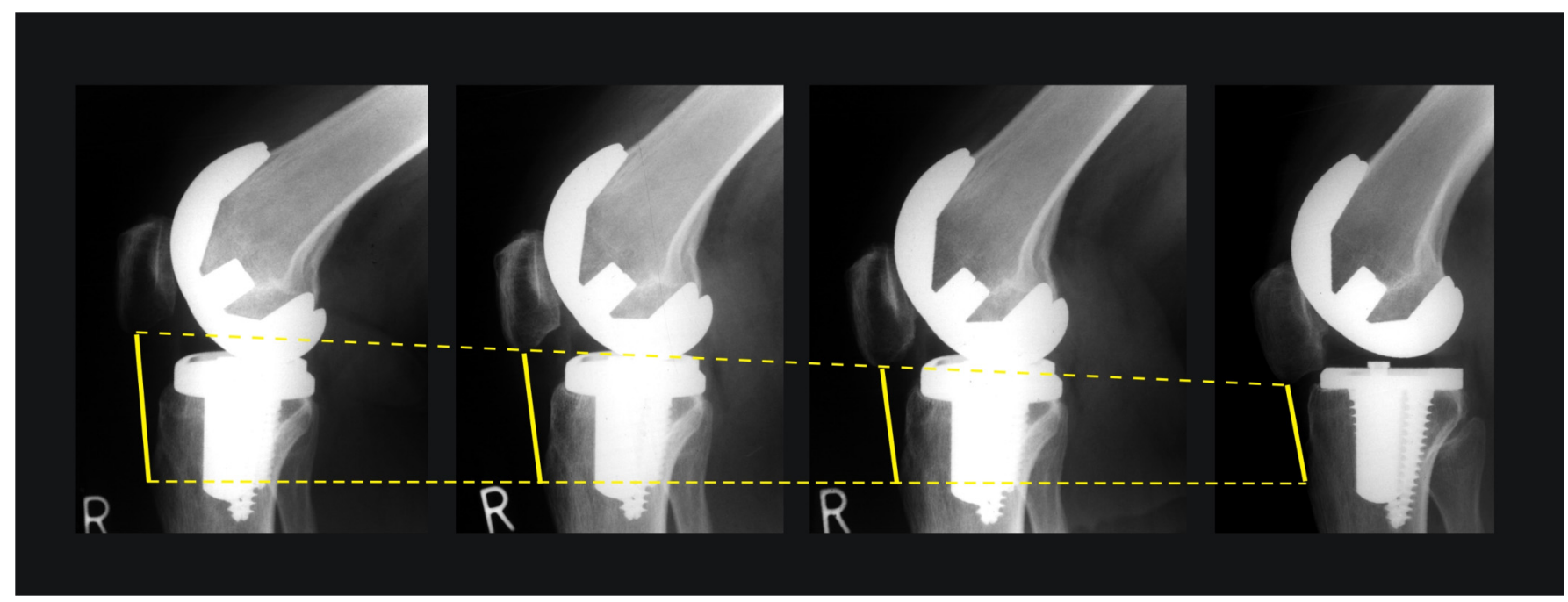

Fig. (1). Development of patella infera. 18 months postoperatively, the patella contacts the PE inlay at $60^{\circ}$ flexion.

ligament, leads to its fibrosis and shortening. However, it cannot be ruled out that a biomechanical conflict that requires the performance of lateral release traumatizes the patellar ligament itself. This interpretation is supported by the investigations of Wojtys, who sees excessive scar formation within the context of arthrofibrosis as the cause of patella infera [9]. However, this causal chain can also be reversed, as a low patella has an unfavorable biomechanical effect and itself leads to restricted motion and incorrect weight-bearing, which can result in a chronic inflammatory reaction $[10,11]$.

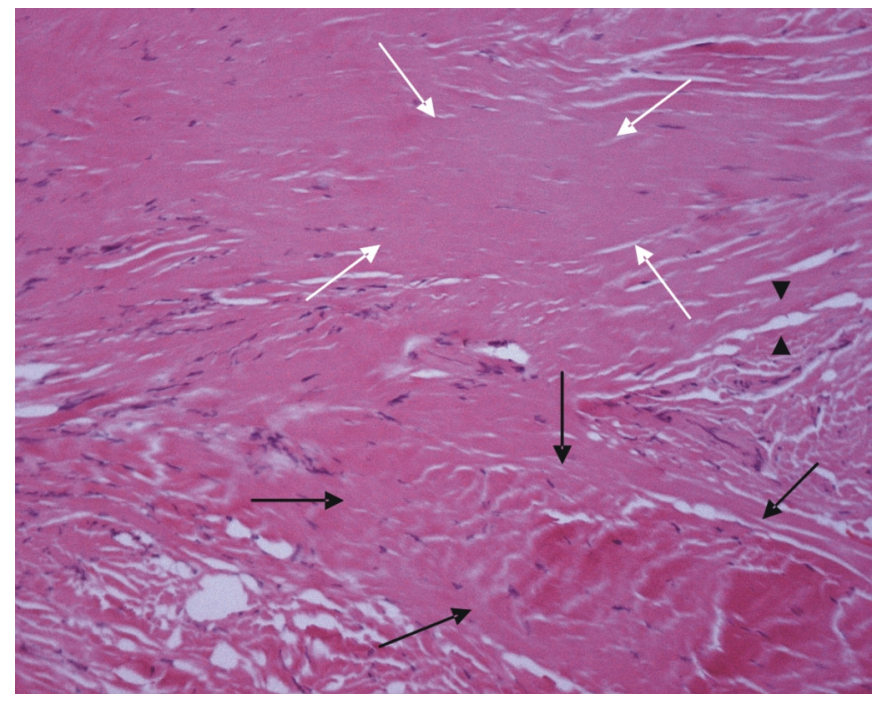

Fig. (2). Section through a biopsy from the patellar ligament, with only partially retained ligament structures in the form of taut collagenic connective tissue (black arrows). The majority of the sample shows degenerative changes, such as maceration of the fibers, the absence of fibrocytes (white arrows) and small foci of lipomatous transformation (arrow tips). Connective tissue proliferation cannot be seen (hematoxylin-eosin, magnification 200x).

In the present case, the intraoperative site showed a correct implantation and centrally running patella, while lateral release had not been performed. We decided against a proximalization of the tuberosity, in order not to reduce the

perfusion of the already shortened patellar ligament [12]. The use of a partially coupled system in this case had the advantage, in our view, that a microtraumatization of Hoffa's body and the patellar ligament on the basis of an impingement by the femoral component can be ruled out, as ap-translation of the components to each other is not possible. The long-term outcome of a partially coupled endoprosthesis is, however, to be observed critically with regard to the high degree of activity of young patients.

In the literature, two different mechanisms of action of the HI virus on wound healing are discussed. On the one hand, an increased release of oxygen radicals by neutrophilic granulocytes occurs, which impairs collagen synthesis and is held responsible for the coincidence of HIV and Dupuytren's contracture $[7,8]$. On the other hand, a reduction in CD4positive lymphocytes in peripheral blood is pathognomonic for HIV infection. T-helper-cells have a chemotactic effect, promote the proliferation of fibroblasts and also influence collagen synthesis $[6,13]$. The present histological picture, which shows degenerative changes in the sense of a maceration of collagen fibers, the loss of fibrocytes and small foci of lipomatous transformation (Fig. 2), might therefore be explained by a lack of these T-cell functions.

The present findings led us to the hypothesis that there is a connection between the patient's HIV infection and the development of the patella infera. The case presented makes it possible to understand failures experienced in HIV positive patients, despite the correct implantation of a total knee replacement. As a result of its low prevalence of less than $1 \%$ in the industrialized countries (WHO 2002), the HI virus cannot explain the occurrence of patella infera after total knee arthroplasty in all cases. However, on the basis of the impaired collagen synthesis and wound healing of an HIVpositive patient, a lower tolerance of the patellar ligament towards microtraumatization and reduced perfusion are conceivable causes of patella infera. We therefore conclude that a particularly soft-tissue sparing surgical method is indicated in the presence of an HIV infection. In this context, discussion in the literature has suggested operating without a 
tourniquet [14], using a subvastus approach [15, 16], doing without lateral release and preserving Hoffa's body [17].

(a)

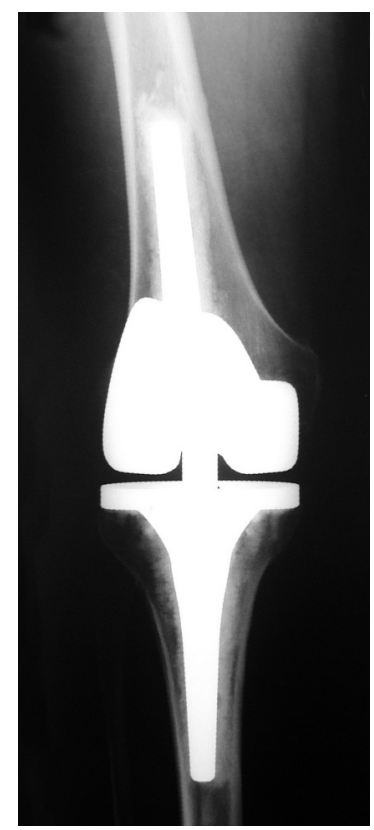

Fig. (3). Postoperative image after change to a partially coupled prosthesis and distalization of the joint line by $5 \mathrm{~mm}$.

\section{REFERENCES}

[1] Grelsamer RP. Patella baja after total knee arthroplasty: is it really patella baja? J Arthroplasty 2002; 17(1): 66-9.

[2] Weale AE, Murray DW, Newman JH, Ackroyd CE. The length of the patellar tendon after unicompartmental and total knee replacement. J Bone Joint Surg Br 1999; 81(5): 790-5.

[3] Kaper BP, Bourne RB, Rorabeck CH, Macdonald SJ. Patellar infera after high tibial osteotomy. J Arthroplasty 2001; 16(2): 16873 .

[4] Wright JM, Heavrin B, Begg M, Sakyrd G, Sterett W. Observations on patellar height following opening wedge proximal tibial osteotomy. Am J Knee Surg 2001; 14(3): 163-73.

[5] Morshed S, Ries MD. Patella infera after nonoperative treatment of a patellar fracture. A case report. J Bone Joint Surg Am 2002; 84-A (6): 1018-21.

[6] Davis PA, Wastell C. A comparison of biomechanical properties of excised mature scars from HIV patients and non-HIV controls. Am J Surg 2000; 180(3): 217-22.

[7] Bower M, Nelson M, Gazzard BG. Dupuytren's contractures in patients infected with HIV. BMJ 1990; 300(6718): 164-5.

[8] Murrell GA. An insight into Dupuytren's contracture. Ann R Coll Surg Engl 1992; 74(3): 156-60.

[9] Wojtys EM, Oakes B, Lindenfeld TN, Bach BR Jr. Patella infera syndrome: an analysis of the patellar tendon pathology. Instr Course Lect 1997; 46: 241-50.

[10] Ahmad CS, Kwak SD, Ateshian GA, Warden WH, Steadman JR, Mow VC. Effects of patellar tendon adhesion to the anterior tibia on knee mechanics. Am J Sports Med 1998; 26(5): 715-24.

[11] Meyer SA, Brown TD, Pedersen DR, Albright JP. Retropatellar contact stress in simulated patella infera. Am J Knee Surg 1997; 10(3): 129-38.

[12] Kanamiya T, Naito M, Ikari N, Hara M. The effect of surgical dissections on blood flow to the tibial tubercle. J Orthop Res 2001; 19(1): 113-6.

[13] Barbul A. Immune aspects of wound repair. Clin Plast Surg 1990; 17(3): 433-42.

[14] Clarke MT, Longstaff L, Edwards D, Rushton N. Tourniquetinduced wound hypoxia after total knee replacement. J Bone Joint Surg $\operatorname{Br} 2001 ; 83(1)$ : 40-4.

[15] Hofmann AA, Plaster RL, Murdock LE. Subvastus (Southern) approach for primary total knee arthroplasty. Clin Orthop 1991; 269: 70-7.

[16] Roysam GS, Oakley MJ. Subvastus approach for total knee arthroplasty: a prospective, randomized, and observer-blinded trial. J Arthroplasty 2001; 16(4): 454-7.

[17] Hughes SS, Cammarata A, Steinmann SP, Pellegrini VD Jr. Effect of standard total knee arthroplasty surgical dissection on human patellar blood flow in vivo: an investigation using laser Doppler flowmetry. J South Orthop Assoc 1998; 7(3): 198-04.

(C) Matziolis et al.; Licensee Bentham Open.

This is an open access article licensed under the terms of the Creative Commons Attribution Non-Commercial License (http://creativecommons.org/licenses/by$\mathrm{nc} / 3.0 /$ ) which permits unrestricted, non-commercial use, distribution and reproduction in any medium, provided the work is properly cited. 